\title{
Direct imaging of the coexistence of ferromagnetism and superconductivity at the $\mathrm{LaAlO}_{3} / \mathrm{SrTiO}_{3}$ interface
}

\author{
Julie A. Bert ${ }^{1}$, Beena Kalisky ${ }^{1}$, Christopher Bell' ${ }^{1}$ Minu Kim ${ }^{1,2}$, Yasuyuki Hikita ${ }^{1}$, Harold Y. Hwang ${ }^{1,2}$ \\ and Kathryn A. Moler ${ }^{1 \star}$
}

$\mathrm{LaAlO}_{3}$ and $\mathrm{SrTiO}_{3}$ are insulating, non-magnetic oxides, yet the interface between them exhibits a two-dimensional electron system with high electron mobility ${ }^{1}$, superconductivity at low temperatures ${ }^{2-6}$ and electric-field-tuned metal-insulator and superconductor-insulator phase transitions ${ }^{3,6-8}$. Bulk magnetization and magnetoresistance measurements also indicate some form of magnetism depending on preparation conditions $^{5,9-11}$ and a tendency towards nanoscale electronic phase separation ${ }^{10}$. Here we use local imaging of the magnetization and magnetic susceptibility to directly observe a landscape of ferromagnetism, paramagnetism and superconductivity. We find submicrometre patches of ferromagnetism in a uniform background of paramagnetism, with a non-uniform, weak diamagnetic superconducting susceptibility at low temperature. These results demonstrate the existence of nanoscale phase separation as indicated by theoretical predictions based on nearly degenerate interface sub-bands associated with the Ti orbitals ${ }^{12,13}$. The magnitude and temperature dependence of the paramagnetic response indicate that the vast majority of the electrons at the interface are localized ${ }^{14}$, and do not contribute to transport measurements ${ }^{3,6,7}$. In addition to the implications for magnetism, the existence of a two-dimensional superconductor at an interface with highly broken inversion symmetry and a ferromagnetic landscape in the background indicates the potential for exotic superconducting phenomena.

Coexistence of ferromagnetism and superconductivity in nature is rare $^{15-19}$. The $\mathrm{LaAlO}_{3} / \mathrm{SrTiO}_{3}$ (LAO/STO) interface is a new system for studying this coexistence. LAO and STO are both perovskite band insulators with no magnetic order in their bulk form. For $\mathrm{LAO}$ grown on the $\mathrm{TiO}_{2}$-terminated STO substrate, a high-mobility electron gas was observed at the interface ${ }^{1}$. Electronic reconstruction, driven by the polar/nonpolar interface, is thought to move charge from the LAO layers across the interface into the STO, causing an effective electronic doping responsible for the observed conductivity ${ }^{1}$. The interplay of this effect with oxygen vacancies and structural changes ${ }^{20}$, and the relative contribution of these three effects to the carrier concentration, remains a subject of debate. Significant variability in the physical properties in similar samples indicates that the ground state of this interface system is sensitive to small changes in growth conditions. Superconductivity ${ }^{2-5}$ and features interpreted as interface magnetism ${ }^{5,9,10}$ have been independently observed at the LAO/STO interface through transport and bulk magnetization measurements. One recent study inferred the existence of both ferromagnetism and superconductivity in the same sample from hysteresis in magnetoresistance transport measurements ${ }^{5}$.

We use a scanning superconducting quantum interference device (SQUID) with micrometre-scale spatial resolution to image three samples down to $20 \mathrm{mK}$ (see Methods). Our SQUID sensor can concurrently measure the static magnetic fields generated by the sample (magnetometry) and the susceptibility of the sample to a small locally applied a.c. magnetic field (susceptometry). Figure 1a,c shows magnetometry and susceptometry images of an LAO/STO interface. The ferromagnetic landscape appears as many static spatially separated dipoles that show no temperature dependence over the measured temperature range. The superconductivity is spatially inhomogeneous and weak, with a critical temperature $T_{\mathrm{c}}=100 \mathrm{mK}$ (Fig. 1e), above which a temperature-dependent paramagnetic response is apparent (Fig. 1e inset). In contrast, a $\delta$-doped STO sample ${ }^{21}$ has relatively uniform two-dimensional superconductivity, no magnetic order and no apparent paramagnetic response above $T_{\mathrm{c}}$ (Fig. 1b,d,f), although the expected paramagnetic signal at $T_{\mathrm{c}}=300 \mathrm{mK}$ is close to our noise floor.

The diamagnetic susceptibility from the LAO/STO interface is an order of magnitude smaller than that of the $\delta$-doped $\mathrm{SrTiO}_{3}$ or $\left(\mathrm{Ba}_{0.9} \mathrm{Nb}_{0.1} \mathrm{CuO}_{2+x}\right)_{m} /\left(\mathrm{CaCuO}_{2}\right)_{n}$, another two-dimensional superconductor $^{22}$. The susceptometry signal is generated by superconducting electrons that screen the local applied field and is related to the local density of electrons in the superconducting condensate. The superfluid density is usually quantified by the magnetic penetration depth, $\lambda$ (refs 23,24$)$. In a two-dimensional superconductor with thickness $d \ll \lambda$, the screening currents are confined in the vertical direction, which generates a modified penetration depth known as the Pearl length, $\Lambda=2 \lambda^{2} / d$. The low-temperature Pearl length in the $\delta$-doped STO sample was $650 \mu \mathrm{m}$ on the basis of fits to formulas for the height dependence of the susceptometry from refs 24,25 . This formula should not quantitatively describe the data for the LAO/STO interface owing to the lateral inhomogeneities, but the susceptibility signal from a uniform two-dimensional superconductor scales as $1 / \Lambda$ for large $\Lambda$, implying an $\sim 8 \mathrm{~mm}$ Pearl length in the LAO/STO.

The $T_{\mathrm{c}}$ variation between two measurement positions on the LAO/STO sample (Fig. 1e) is about 10\%. However, the lateral variation of the susceptometry is large: $84 \%$ of the total response, compared with just $12 \%$ in the $\delta$-doped STO, and less than $1 \%$ in most bulk superconductors ${ }^{26}$. The largely inhomogeneous superconducting and ferromagnetic response may indicate proximity to a first-order phase transition. Although both

\footnotetext{
${ }^{1}$ Stanford Institute for Materials and Energy Science, Stanford University, Stanford, California 94305, USA, ${ }^{2}$ Department of Advanced Materials Science, University of Tokyo, Kashiwa, Chiba 277-8561, Japan. *e-mail: kmoler@stanford.edu.
} 
a

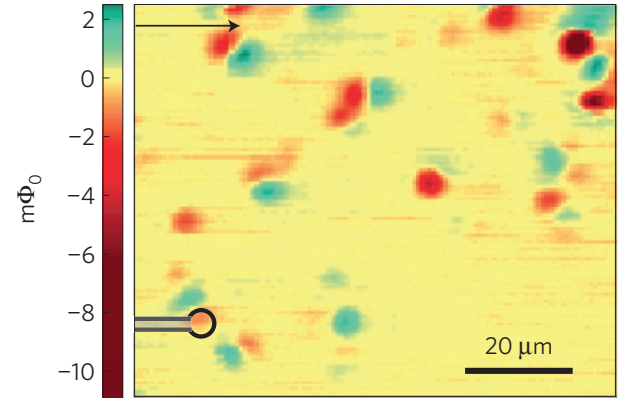

c

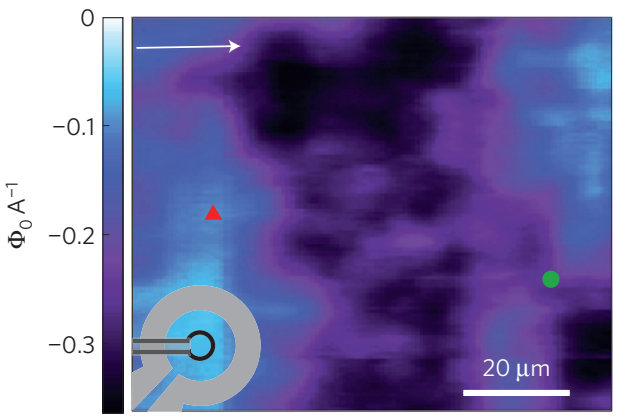

e

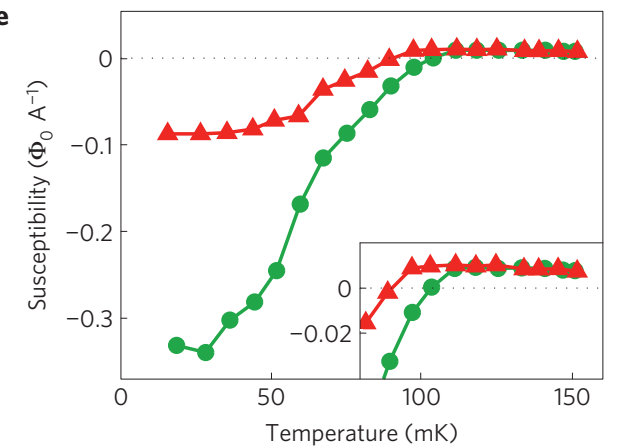

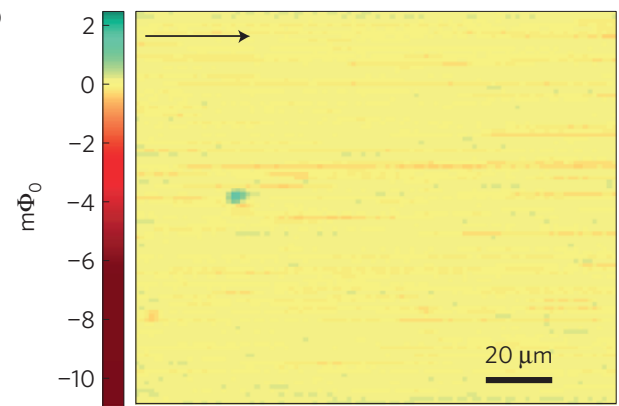

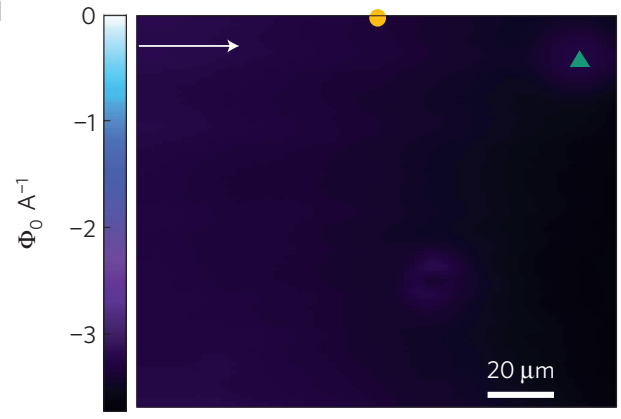

$\mathbf{f}$

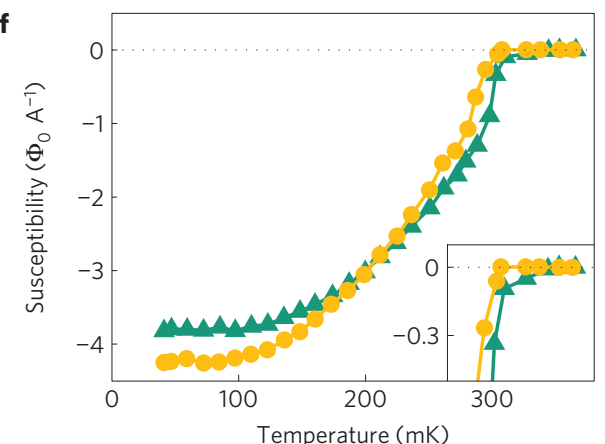

Figure 1 | Comparison of SQUID images on LAO/STO and $\boldsymbol{\delta}$-doped STO samples. a, LAO/STO magnetometry image mapping the ferromagnetic order. Inset: Scale image of the SQUID pick-up loop used to sense magnetic flux. b, $\delta$-doped STO magnetometry image showing no ferromagnetic order. c, LAO/STO susceptometry image mapping the superfluid density at $40 \mathrm{mK}$. Inset: Scale image of the SQUID pick-up loop and field coil. d, $\delta$-doped STO susceptometry image mapping the superfluid density at $82 \mathrm{mK}$. e, The temperature dependence of the susceptibility taken at the two positions indicated in c. f, The temperature dependence of the susceptibility taken at the two positions indicated in $\mathbf{e}$. The arrow on each scan shows the scan fast axis and the SQUID orientation.

magnetism and superconductivity are present at the interface in the LAO/STO sample, Fig. 1a,c does not show a direct correlation between the inhomogeneity of the superconducting state and the distribution of magnetic regions.

The ferromagnetism appears as magnetic dipoles in Figs 1a and $2 \mathrm{a}$, mostly separated from one another by micrometres, with many extra dipoles that do not show up visually in these images but are still above our noise threshold (Fig. 2a insets). We analysed six $70 \times 80 \mu \mathrm{m}$ high-resolution magnetometry scans, including the one shown in Fig. 2a, finding 144 dipoles above our noise floor and fitting each one to a point-dipole model to determine its total moment and orientation (Fig. 2b-d). The histogram of the dipole moments shows a clear exponential distribution of dipole moments with a few large $\left(\sim 1 \times 10^{8} \mu_{\mathrm{B}}\right)$ dipoles and substantially more smaller dipoles down to the limit of our noise. This trend indicates that there may be even more dipoles with moments below the sensitivity of our SQUID.

Most of the dipoles lie in plane, as expected from the shape anisotropy of the interface, with apparently randomly distributed azimuthal angles indicating no alignment or net magnetization. This observation is consistent with cooling the sample in zero field. The point-dipole approximation is not as good for some dipoles, particularly the ones with the largest moments, indicating that they are not point-like but are instead ferromagnetic patches that extend over an area comparable to the SQUID's $3 \mu \mathrm{m}$ pick-up loop. The dipoles were stable throughout the duration of the cool-down (about 1 month) and were insensitive to temperature changes from $20 \mathrm{mK}$ through the superconducting critical temperature and up to $4.2 \mathrm{~K}$. Further SQUID measurements in a separate variable-temperature cryostat showed that the dipole size and orientation remained unchanged between $4.2 \mathrm{~K}$ and our maximum measurement temperature of $60 \mathrm{~K}$. In addition, we measured a second sample with 10 unit cells (u.c.) of $\mathrm{LAO}$ grown on a $\mathrm{TiO}_{2}-$ terminated surface that had patterned Hall bars (Methods). This second 10 u.c. LAO/STO sample had many fewer dipoles-none in some regions. The variability in the size of the moment may be related to the variability of physical properties in nominally identical samples in this system.

We did not observe dipoles in the magnetometry signal on the $\delta$-doped STO sample (Fig. 1b). As both the $\delta$-doped STO sample and the LAO/STO samples use the same commercially available STO substrates, the absence of dipoles on the $\delta$-doped STO sample rules out magnetic impurities in the substrate. This observation is corroborated by the scan height extracted from 
a
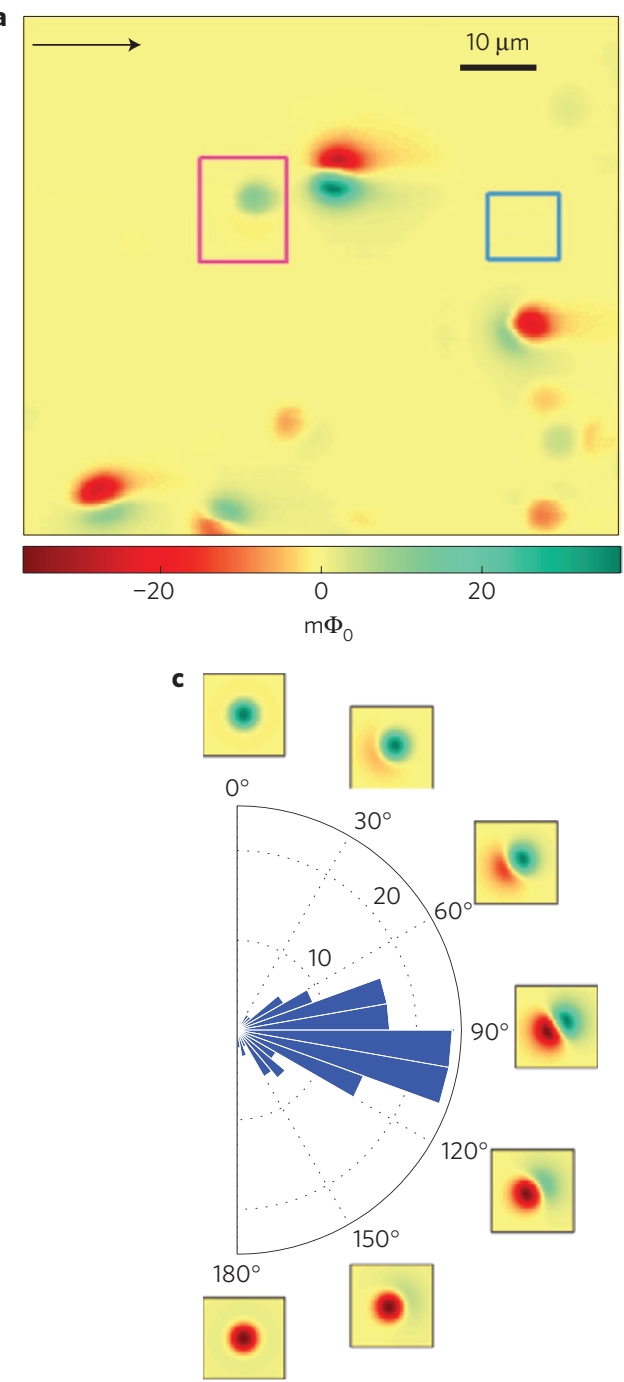
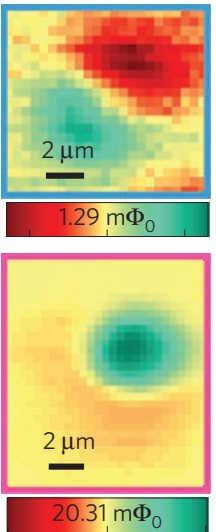

b

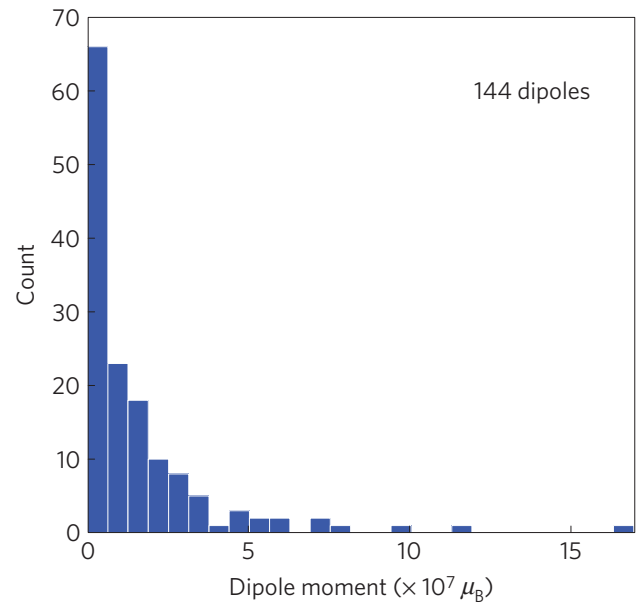

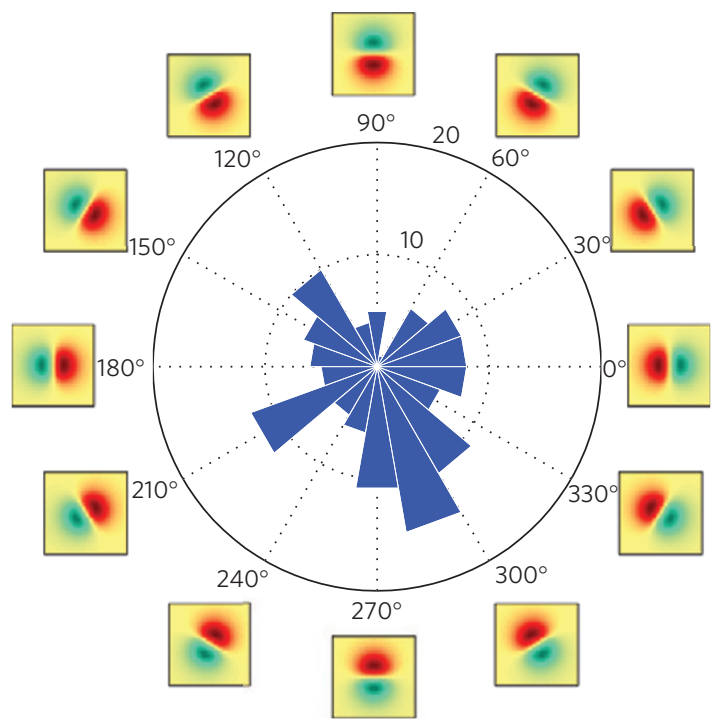

Figure $\mathbf{2}$ | Analysis of the dipole distribution. a, Magnetometry scan showing ferromagnetic dipoles. The arrow shows the scan fast axis and the SQUID orientation. Insets: Individual dipoles from the areas indicated in the larger image. b-d, Histograms of the moment and orientation of 144 dipoles taken from six large-area scans similar to the one shown in $\mathbf{a}$. $\mathbf{b}$. The magnetic moment of each dipole in Bohr magnetons, $\mu_{B}$. $\mathbf{c}$, The inclination angle from the normal to the sample surface (an inclination angle of $90^{\circ}$ means the dipole lies in the plane of the interface). $\mathbf{d}$, The azimuthal angle with respect to the scan's $x$ axis.
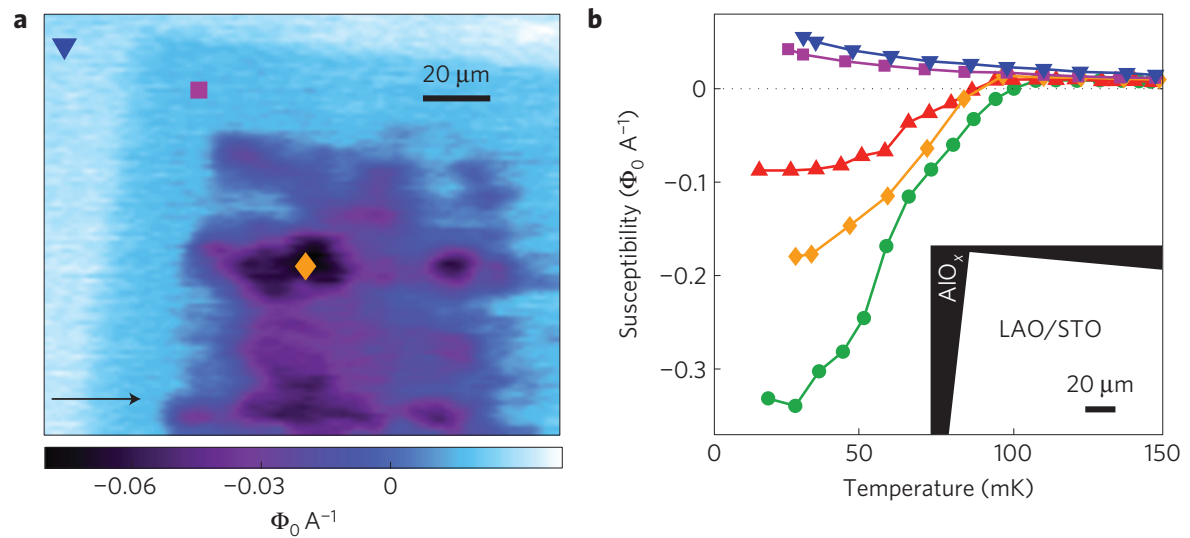

Figure 3 | Paramagnetic signal on patterned LAO/STO sample. a, Susceptometry scan on the patterned sample at $87 \mathrm{mK}$. A suppression of the diamagnetic susceptibility is visible near the edge of the pattern. The susceptibility response in this area has a paramagnetic sign as indicated in the susceptibility-versus-temperature plot. The arrow indicates the scan fast axis and the SQUID orientation. b, Susceptibility-versus-temperature data from three positions on the patterned LAO/STO shown in $\mathbf{a}$. The red triangles and green circles are data reproduced from the unpatterned LAO/STO sample shown in Fig. 1a. Inset: The outline of the $\mathrm{AlO}_{x}$ patterning associated with the susceptometry scan. 
Table 1 | Table of electron densities.

\begin{tabular}{lllll} 
& \multicolumn{1}{c}{ LAO/STO } & Patterned & \multicolumn{1}{c}{$\begin{array}{c}\text {-doped STO } \\
\text { Unpatterned }\end{array}$} \\
\cline { 2 - 3 } & Literature & Unpatterned & - & - \\
Polar catastrophe & $3.2 \times 10^{14}$ & - & - & $4.7 \times 10^{13}($ ref. 21$)$ \\
Hall effect ${ }^{3,6,7}$ & $1-4 \times 10^{13}$ & - & $1-5 \times 10^{14}$ & - \\
Paramagnetic & - & $1-5 \times 10^{14}$ & $<2 \times 10^{11 \star}$ & $<1 \times 10^{11 \star}$ \\
Dipole moment $\left(\mu_{\mathrm{B}}\right)$ & - & $0.4-1.1 \times 10^{13}$ & $0-1 \times 10^{12}$ & $1-1.4 \times 10^{13}$ \\
Superfluid density & - & $0.3-2 \times 10^{12}$ & & \\
\hline
\end{tabular}

Electron densities extracted from Hall measurements, and measurement of the ferromagnetic, superconducting and paramagnetic signals for all three samples. All quantities are given in $\mathrm{cm}^{-2}$. The - symbol means this measurement is not applicable. ${ }^{\star}$ Dipoles were too sparse and inhomogeneously distributed to extract a statistically significant areal density.

the dipole fits, indicating that the dipoles are within a few micrometres of the surface.

In addition to the ferromagnetic order, the two LAO/STO samples measured at low temperature show paramagnetism above the superconducting critical temperature $T_{\mathrm{c}}$ (Fig. 1e inset, Fig. 3). In the case of the patterned LAO/STO sample, which did not show many ferromagnetic dipoles, we observed regions near the Hall-bar edges where the superconductivity was suppressed and the paramagnetism remained down to the lowest measured temperatures. The paramagnetic signal decreases with increasing temperature, indicating a Curie law. The $1 / T$ dependence and the paramagnetic sign indicate that the susceptibility signal originates from localized spins.

We can estimate the electron density associated with the ferromagnetic, diamagnetic and paramagnetic signals. We determine the number of ferromagnetic electrons by adding the moments of all the dipoles in the histogram, yielding $7.3 \pm 3.4 \times 10^{12} \mu_{\mathrm{B}} \mathrm{cm}^{-2}$. This estimate is a lower bound, because any dipoles that are below the sensitivity of our sensor or whose moments cancelled owing to the random distribution of alignments have not been included in this total. We use the Pearl length to find the density of superconducting electrons, $n_{\mathrm{s}}=2 m^{*} / \mu_{0} e^{2} \Lambda$, where $e$ is the elementary charge and $\mu_{0}$ is the permeability of free space. Using $m^{*}=1.45 m_{e}$ (ref. 27), we find $n_{\mathrm{s}} \approx 1 \times 10^{12} \mathrm{~cm}^{-2}$ in the regions of high superfluid density and $n_{\mathrm{s}} \approx 3 \times 10^{11} \mathrm{~cm}^{-2}$ in the regions of low superfluid density. We quantify the paramagnetic signal by using an appropriate model for our sensor to convert our measured susceptibility, $\phi$, to the dimensionless susceptibility, $\chi$, for a layer of spins in a thickness $d$. Using $\chi d=22 \mu \mathrm{m}\left(\mathrm{mA} / \Phi_{0}\right) \phi$ (ref. 28) and comparing $\chi$ with the Curie expression, $\chi=\mu_{0} n_{3 \mathrm{D}}\left(g \mu_{\mathrm{B}}\right)^{2} J(J+1) / 3 k_{\mathrm{B}} T$ with $g=2$ and $J=1 / 2$, yields a two-dimensional spin density of $4.4 \times 10^{14} \mathrm{~cm}^{-2}$, with large error bars due to uncertainty in the geometrical parameters. We compare our estimates with the electron densities predicted by the polar catastrophe, $3 \times 10^{14} \mathrm{~cm}^{-2}$, and seen in Hall measurements ${ }^{3,6,7}, 1-4 \times 10^{13} \mathrm{~cm}^{-2}$ (Table 1 ). The densities of magnetic and superconducting electrons are respectively one and two orders of magnitude lower than the polar catastrophe density, but the paramagnetic spin density shows surprising agreement within error.

Density functional calculations of the electronic structure in LAO/STO predict the presence of multiple nearly degenerate subbands that result in separate charge carriers ${ }^{12}$. Magnetism was also predicted at the n-type LAO/STO interface from alignment of extra electrons in the Ti orbitals ${ }^{13}$. Transport measurements, which probe delocalized electrons, have measured electron densities significantly lower than predictions from the polar catastrophe. Our measurements indicate that these missing electrons may be present but localized, and contribute to the magnetic signal.

The observation of ferromagnetism and superconductivity at the LAO/STO interface opens exciting possibilities for studying the interplay of these normally incompatible states. Tuning the carriers with a gate voltage may add even more richness to the system, by coincidentally studying how adding or removing carriers affects the superconducting, ferromagnetic and paramagnetic signals.

Note added in proof: Recent torque magnetometry in a similar sample ${ }^{29}$ shows magnetic ordering consistent with interface magnetism. The variety of details in reports of magnetism ${ }^{9-11,29}$, including our observation of a landscape (rather than a homogeneous phase), support the exciting possibility that multiple states are important in this engineered interface.

\section{Methods}

The two LAO/STO samples used in the low-temperature study were prepared by growing 10 u.c. of $\mathrm{LaAlO}_{3}$ on commercial $\mathrm{TiO}_{2}$-terminated $001 \mathrm{STO}$ substrates. The patterned sample had an $\mathrm{AlO}_{x}$ hard mask, which defined Hall bars. The $\mathrm{LaAlO}_{3}$ was deposited at $800^{\circ} \mathrm{C}$ with an oxygen partial pressure of $10^{-5} \mathrm{mbar}$, after a pre-anneal at $950^{\circ} \mathrm{C}$ with an oxygen partial pressure of $5 \times 10^{-6} \mathrm{mbar}$ for $30 \mathrm{~min}$. The samples were cooled to $600{ }^{\circ} \mathrm{C}$ and annealed in a high-pressure oxygen environment ( 0.4 bar) for one hour ${ }^{6}$

A $\delta$-doped STO sample was also studied at low temperatures. It was grown in an atmosphere of less than $10^{-8}$ torr oxygen at $1,200^{\circ} \mathrm{C}$. $\mathrm{Nb}$ dopants were confined to a $5.9 \mathrm{~nm}$ layer, and further $100 \mathrm{~nm}$ cap and buffer layers of STO were grown above and below the doped region. The sample was annealed in situ at $900^{\circ} \mathrm{C}$ under an oxygen partial pressure of $10^{-2}$ torr for $30 \mathrm{~min}$ (ref. 21).

Measurements were made by scanning SQUID in a dilution refrigerator ${ }^{30,31}$ The SQUID has a $3 \mu \mathrm{m}$ pick-up loop, centred in a single-turn field coil. Static magnetism (magnetometry) in the sample is probed by recording the flux through the SQUID pick-up loop as a function of position. Applying an a.c. current in the field coil produces a local magnetic field. The local susceptibility (susceptometry) of the sample to the applied field is detected by the pick-up loop in a lock-in measurement.

Received 10 May 2011; accepted 2 August 2011; published online 4 September 2011

\section{References}

1. Ohtomo, A. \& Hwang, H. Y. A high-mobility electron gas at the $\mathrm{LaAlO}_{3} / \mathrm{SrTiO}_{3}$ heterointerface. Nature 427, 423-426 (2004).

2. Reyren, N. et al. Superconducting interfaces between insulating oxides. Science 317, 1196-1199 (2007).

3. Caviglia, A. D. et al. Electric field control of the $\mathrm{LaAlO}_{3} / \mathrm{SrTiO}_{3}$ interface ground state. Nature 456, 624-627 (2008)

4. Ben Shalom, M., Sachs, M., Rakhmilevitch, D., Palevski, A. \& Dagan, Y. Tuning spin-orbit coupling and superconductivity at the $\mathrm{SrTiO}_{3} / \mathrm{LaAlO}_{3}$ interface: A magnetotransport study. Phys. Rev. Lett. 104, 126802 (2010).

5. Dikin, D. A. et al. Coexistence of superconductivity and ferromagnetism in two dimensions. Phys. Rev. Lett. 107, 056802 (2011).

6. Bell, C. et al. Dominant mobility modulation by the electric field effect at the $\mathrm{LaAlO}_{3} / \mathrm{SrTiO}_{3}$ interface. Phys. Rev. Lett. 103, 226802 (2009).

7. Thiel, S., Hammerl, G., Schmehl, A., Schneider, C. W. \& Mannhart, J. Tunable quasi-two-dimensional electron gases in oxide heterostructures. Science 313, 1942-1945 (2006).

8. Cen, C. et al. Nanoscale control of an interfacial metal-insulator transition at room temperature. Nature Mater. 7, 298-302 (2008).

9. Brinkman, A. et al. Magnetic effects at the interface between non-magnetic oxides. Nature Mater. 6, 493-496 (2007).

10. Ariando, et al. Electronic phase separation at the $\mathrm{LaAlO}_{3} / \mathrm{SrTiO}_{3}$ interface. Nature Commun. 2, 188 (2011)

11. Seri, S. \& Klein, L. Antisymmetric magnetoresistance of the $\mathrm{SrTiO}_{3} / \mathrm{LaAlO}_{3}$ interface. Phys. Rev. B 80, 180410 (2009). 
12. Popović, Z. S., Satpathy, S. \& Martin, R. M. Origin of the two-dimensional electron gas carrier density at the $\mathrm{LaAlO}_{3}$ on $\mathrm{SrTiO}_{3}$ interface. Phys. Rev. Lett. 101, 256801 (2008).

13. Pentcheva, R. \& Pickett, W. E. Charge localization or itineracy at $\mathrm{LaAlO}_{3} / \mathrm{SrTiO}_{3}$ interfaces: Hole polarons, oxygen vacancies, and mobile electrons. Phys. Rev. B 74, 035112 (2006).

14. Sing, M. et al. Profiling the interface electron gas of $\mathrm{LaAlO}_{3} / \mathrm{SrTiO}_{3}$ heterostructures with hard X-ray photoelectron spectroscopy. Phys. Rev. Lett. 102, 176805 (2009).

15. Aoki, D. et al. Coexistence of superconductivity and ferromagnetism in URhGe. Nature 413, 613-616 (2001).

16. Saxena, S. S. et al. Superconductivity on the border of itinerant-electron ferromagnetism in $\mathrm{UGe}_{2}$. Nature 406, 587-592 (2000).

17. Bulaevskii, L. N., Buzdin, A. I., Kulic, M. L. \& Panjukov, S. V. Coexistence of superconductivity and magnetism. Theoretical predictions and experimental results. Adv. Phys. 34, 175-261 (1985)

18. Buzdin, A. I. Proximity effects in superconductor-ferromagnet heterostructures. Rev. Mod. Phys. 77, 935-976 (2005).

19. Tallon, J. et al. Coexisting ferromagnetism and superconductivity in hybrid rutheno-cuprate superconductors. Appl. Supercond. 9, 1696-1699 (1999).

20. Huijben, M. et al. Structure property relation of $\mathrm{SrTiO}_{3} / \mathrm{LaAlO}_{3}$ interfaces. Adv. Mater. 21, 1665-1677 (2009).

21. Kozuka, Y. et al. Two-dimensional normal-state quantum oscillations in a superconducting heterostructure. Nature 462, 487-490 (2009).

22. Tafuri, F., Kirtley, J. R., Medaglia, P. G., Orgiani, P. \& Balestrino, G. Magnetic imaging of Pearl vortices in artificially layered $\left(\mathrm{Ba}_{0.9} \mathrm{Nd}_{0.1} \mathrm{CuO}_{2+x}\right)_{m} /\left(\mathrm{CaCuO}_{2}\right)_{n}$ systems. Phys. Rev. Lett. 92, 157006 (2004)

23. Luan, L. et al. Local measurement of the penetration depth in the pnictide superconductor $\mathrm{Ba}\left(\mathrm{Fe}_{0.95} \mathrm{Co}_{0.05}\right)_{2} \mathrm{As}_{2}$. Phys. Rev. B 81, 100501 (2010).

24. Hicks, C. W. et al. Evidence for a nodal energy gap in the iron-pnictide superconductor LaFePO from penetration depth measurements by scanning SQUID susceptometry. Phys. Rev. Lett. 103, 127003 (2009).

25. Kogan, V. G. Meissner response of anisotropic superconductors. Phys. Rev. B 68, 104511 (2003).
26. Kalisky, B. et al. Stripes of increased diamagnetic susceptibility in underdoped superconducting $\mathrm{Ba}\left(\mathrm{Fe}_{1-x} \mathrm{Co}_{x}\right)_{2} \mathrm{As}_{2}$ single crystals: Evidence for an enhanced superfluid density at twin boundaries. Phys. Rev. B 81, 184513 (2010).

27. Caviglia, A. D. et al. Two-dimensional quantum oscillations of the conductance at $\mathrm{LaAlO}_{3} / \mathrm{SrTiO}_{3}$ interfaces. Phys. Rev. Lett. 105, 236802 (2010).

28. Bluhm, H., Bert, J. A., Koshnick, N. C., Huber, M. E. \& Moler, K. A. Spinlike susceptibility of metallic and insulating thin films at low temperature. Phys. Rev. Lett. 103, 026805 (2009).

29. Li, L., Richter, C., Mannhart, J. \& Ashoori, R. C. Coexistence of magnetic order and two-dimensional superconductivity at $\mathrm{LaAlO}_{3} / \mathrm{SrTiO}_{3}$ interfaces. Nature Phys. 7, 762-766 (2011).

30. Huber, M. E. et al. Gradiometric micro-SQUID susceptometer for scanning measurements of mesoscopic samples. Rev. Sci. Instrum. 79, 053704 (2008).

31. Björnsson, P. G., Gardner, B. W., Kirtley, J. R. \& Moler, K. A. Scanning superconducting quantum interference device microscope in a dilution refrigerator. Rev. Sci. Instrum. 72, 4153-4158 (2001).

\section{Acknowledgements}

We thank M. Huber for assistance in SQUID design and fabrication. This work was primarily supported by the US Department of Energy, Office of Basic Energy Sciences, Division of Materials Sciences and Engineering under award DE-AC02-76SF00515. B.K. acknowledges support from FENA.

\section{Author contributions}

SQUID measurements: J.A.B. and B.K. Analysis: J.A.B. and B.K. with ideas developed with H.Y.H. and K.A.M. Sample growth: C.B., M.K., Y.H., and H.Y.H. Manuscript preparation: J.A.B., H.Y.H and K.A.M., with input from all co-authors.

\section{Additional information}

The authors declare no competing financial interests. Reprints and permissions information is available online at http://www.nature.com/reprints. Correspondence and requests for materials should be addressed to K.A.M. 